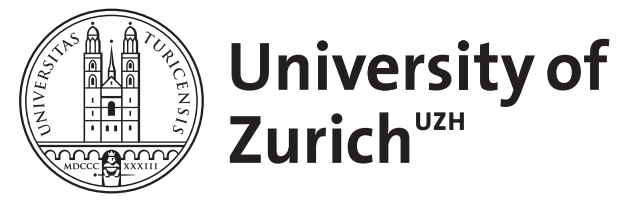

Zurich Open Repository and Archive

University of Zurich

University Library

Strickhofstrasse 39

CH-8057 Zurich

www.zora.uzh.ch

Year: 2010

IV weibelt für berufliche Integration - Goodwill auf schwierigem Terrain

Wolff, Eberhard

Posted at the Zurich Open Repository and Archive, University of Zurich ZORA URL: https://doi.org/10.5167/uzh-40396

Journal Article

Originally published at:

Wolff, Eberhard (2010). IV weibelt für berufliche Integration - Goodwill auf schwierigem Terrain. Schweizerische Ärztezeitung, 91(13/14):560. 


\section{IV weibelt für berufliche Integration - Goodwill auf schwierigem Terrain}

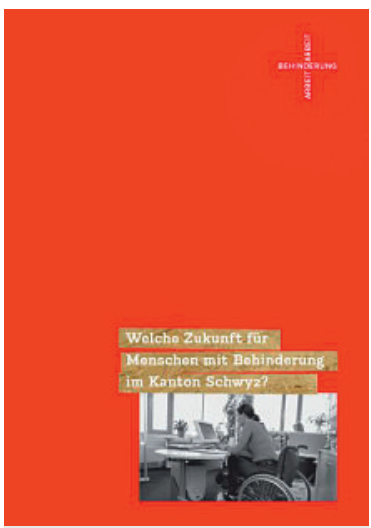

Die Wanderausstellung «50 Jahre IV» der IV-StellenKonferenz hat ihre Schweizerreise angetreten und wird bis Ende 2010 , angereichert durch Rahmenprogramme, meist an Messen gezeigt. Darunter: Luga (Luzern), Ostschweizer Personaltag (St. Gallen), Handicap sur la Fête (Delémont), GEWA (Seewen), HESO (Solothurn). Details unter www.ivsk.ch.

Siehe auch «50 Jahre IV: die Bedeutung des beruflichen Eingliederungsmodells» auf Seite 559.

* PD Dr. rer. soc.

Eberhard Wolff ist Kulturwissenschaftler, Medizinhistoriker und Mitglied der Redaktion Medizingeschichte der Schweizerischen Ärztezeitung.
Wenn es um die Invalidenversicherung (IV) geht, standen in den letzten Jahren die Nullen im Mittelpunkt. Vierzehn Milliarden Franken Defizit, allein 2009 betrug die Neuverschuldung eine Milliarde. Die Sanierung ist in die Wege geleitet, das Stimmvolk akzeptierte hierfür mit knappem Mehr die befristete Erhöhung der Mehrwertsteuer. Auch die IV selber nimmt sich in die Pflicht. Doch sie steht in einem Dilemma. Dreht sie den Ausgabenhahn nur behutsam zu, bleibt zu viel Defizit, dreht sie zu sehr, lautet die Kritik auf das Abschieben Behinderter in andere Sozialwerke oder den Ausschluss ernsthaft Bedürftiger.

Um den Druck dieses Schraubstocks zu mildern, besinnt sich die IV, genauer: der interkantonale Dachverband «IV-Stellen-Konferenz» (IVSK) auf alte Tugenden und intensiviert die Bemühungen um die berufliche Integration von «Menschen mit gesundheitlichen Einschränkungen». Das ist billiger für die IV und die Steuerzahler und obendrein meist im Sinne der Klienten. Wer arbeitet, hat bei uns oft mehr vom Leben. offenbar nicht aus, um Menschen mit Fähigkeiten, aber auch mit Handicaps, im beruflichen Leben unterzubringen. Es sollen Vorurteile abgebaut werden. Viele Kompetenzen von Behinderten würden von der eingeengten Wahrnehmung als «Behinderte» überdeckt. Und wenn man bedenkt, dass auch die als nicht behindert angesehenen Kolleginnen und Kollegen den Betrieben Krankheits- und andere Kosten bereiteten, fallen die Unterschiede im Vergleich nicht mehr so deutlich aus.

So gibt die Ausstellung Idealbeispiele beruflicher Integration, wie eine Pflegefachfrau, die nach einer schweren depressiven Erkrankung im Service eines sozial engagierten Hotels und bei geringerem Arbeitsdruck wieder im Arbeitsleben Fuss gefasst hat. Oder sie präsentiert den sprachbehinderten Mann, der eine Stelle in einer Schreinerei gefunden hat, die ein offenes Ohr für Menschen mit Handicap hat. Das kann funktionieren, auch bei gewinnorientierten Unternehmen. «Trotzdem gibt es noch 95000 Unterneh-

\section{«Finanzielle Anreize reichen offenbar nicht aus, um Menschen mit Fähigkeiten, aber auch mit Handicaps, im beruflichen Leben unterzubringen»}

Ein Nebeneffekt mag sein, das angekratzte Image etwas zu polieren und den sozialpolitischen Debatten, die sich zur Zeit so oft um «Betrug» und «Detektive» drehen, den Horizont zu erweitern. Dabei geht die IVSK sanfter vor als unlängst ihre Kollegen von der Bundes-IV mit der provokativ-umstrittenen Plakatkampagne. Sie erinnern sich: «Behinderte kosten uns nur Geld ...».

Da kommt ein Jubiläum gerade recht. «50 Jahre IV» ist eine Ausstellung, die bald durch die gesamte Schweiz touren wird (siehe Kasten). Vor allem auf Messen soll das Gespräch mit der Bevölkerung und nicht zuletzt mit potentiellen Arbeitgebern gesucht werden. 192000 fehlende Arbeitsplätze für ihre Klienten hat die IV ausgemacht. Finanzielle Anreize reichen men, die bereit wären, Menschen mit Behinderungen anzustellen, und es doch nicht tun», sagt Stefan Ritler, Ex-Präsident der IVSK und ab Mai IV-Chef im Bundesamt für Sozialversicherung. Im Unterschied zu Staaten wie Deutschland kennt die Schweiz nämlich keine Zwangsmassnahmen gegenüber Arbeitgebern.

Jobvermittlung für Menschen mit Handicap, das ist in Zeiten der Krise aber ein delikates Terrain. Wie der Tagesanzeiger kürzlich meldete, machen sich die verschiedenen Sozialstellen im Kampf um die «Nischenarbeitsplätze» zunehmend gegenseitig Konkurrenz (6.1.2010). Ein immer engerer Markt ruft so nach fantasievollen Aktionen.

Eberhard Wolff* 